\title{
JOSEPH JURT
}

\section{Bourdieus Kapital-Theorie}




\section{Bourdieus Kapital-Theorie ${ }^{1}$}

Joseph Jurt

Nachdem sich Pierre Bourdieu während seiner Algerien-Zeit (1956-1960) das soziologische Rüstzeug angeeignet hatte, das ihm erlaubte, diese Gesellschaft im Umbruch zu verstehen, widmete er sich unmittelbar nach seiner Rückkehr nach Frankreich der Bildungssoziologie. Im Zentrum standen dabei die gesellschaftlichen Reproduktionsweisen des Bildungssystems. Das forschungsstrategisch wichtigste Resultat der bildungssoziologischen Untersuchungen war seine Kapital-Theorie und hier namentlich das Konzept des kulturellen Kapitals.

Epistemologisch zentral war für ihn, die Aporien des Subjektivismus, namentlich der Existenz- Philosophie Sartres, und des Objektivismus des strukturalistischen Ansatzes zu überwinden durch eine Theorie der Praxis. Diese praxeologische Erkenntnisweise war nicht die Frucht eines abstrakten Gedankensystems, sondern entstand in und durch die empirischen ethnologischen und soziologischen Untersuchungen der algerischen Gesellschaft, des ländlichen Milieus der Pyrenäen und des Bildungssystems Frankreichs.

Auf der Basis dieser erkenntnistheoretischen Position bildeten sich dann drei zentrale Kategorien aus, die den Ansatz von Bourdieu unverwechselbar prägen: der Begriff des Habitus als Vermittlungsinstanz zwischen Struktur und Subjekt, dann die schon erwähnte Kategorie des Kapitals, das aber nicht bloß als ökonomische Größe gedacht wird. Der Begriff der Gesellschaft wird schließlich ersetzt durch den des sozialen Raums, der sich ausdifferenziert in unterschiedliche $\mathrm{Fel}$ der. Mit diesen drei zentralen Kategorien brachte Bourdieu wieder die Akteure ins Spiel und auch die Geschichtlichkeit, die durch den Strukturalismus ausgeblendet worden waren, ohne jedoch in eine Subjektphilosophie zurückzufallen.

1 Die folgenden Ausführungen beruhen auf dem Abschnitt: Eine andere Ökonomie: die vier Kapitalarten in: Jurt( 2008: 70-90). 


\section{Zum Kapitalbegriff Bourdieus}

Unter dem Begriff des Habitus werden verkörperte Eigenschaften des Sozialen verstanden, Dispositionen, die sich der Sozialisation verdanken und die bestimmte Handlungs- und Wahrnehmungsschemata generieren ${ }^{2}$ Im Handeln werden aber auch Handlungsobjekte geschaffen. Um diese zu bezeichnen, führt Bourdieu den Begriff Kapital ein, den er Marx entlehnt, aber nicht in einem marxistischen Sinn interpretiert. Bourdieu kannte wohl Marx ganz gut, war aber nie Marxist, sondern entnahm Marx einzelne Konzepte, wenn das ihm sinnvoll und nützlich erschien. Er griff auch auf Max Webers Unterscheidung von Klassenlage (der Marktlage auf dem Güter- und Arbeitsmarkt) und Klassenstand (Stellung innerhalb der Hierarchie von Ehre und Prestige) zurück (Bourdieu 1974: 42-74). Das Interesse für die symbolischen Formen teilte er mit Cassirer. Seinen relationellen Ansatz, der sich dem Substantialismus widersetzte, begründete er mit dem Hinweis auf Cassirers Beitrag Substanz und Form, der diesen Ansatz als den Denkmodus der modernen Wissenschaft nannte. Neben Max Weber und Cassirer war Panofsky ein weiterer wichtiger Inspirator von Pierre Bourdieu. Panofsky hatte Analogien zwischen der gotischen Architektur und der scholastischen Philosophie auf kollektive Denkgewohnheiten zurückgeführt, die er Habitus nannte, ein Gestaltungsprinzip, das durch eine Institution geprägt werde: die Schule als verhaltensnormierende Instanz. Diesen Ansatz wandte Bourdieu auf eine ganze Anzahl unterschiedlicher Felder der zeitgenössischen Gesellschaft an (Bourdieu 1974: 125-158). Bourdieu hatte auch Durkheims Idee, dass die soziale Ordnung unser Denken und Handeln bestimmt, weil sie unsere Klassifikationssysteme modelliert, weiter entwickelt (Wacquant 1995: 646-660). Im Unterschied zu Durkheim und Lévi-Strauss stimmte er mit Marx und Max Weber darin überein, dass Sinnbeziehungen auf Machtbeziehungen beruhen, wobei er dem Symbolischen eine Eigenlogik zugesteht.

Die Schulzuschreibungen (Marxist, Durkheimianer, Weberianer) stellen in den Augen Bourdieus ein Haupthindernis für den wissenschaftlichen Fortschritt dar, weil sie verhindern, falsche Antinomien zu überwinden. Bourdieu plädiert für eine „Realpolitik des Begriffs“ (Bourdieu 1992: 40), die sich an einer theoretischen Linie orientiert, die vor einem baren Eklektizismus schützt. Er spricht darum von einem von den genannten Autoren gebildeten „Theorie-Raum“, der den Raum der Möglichkeiten und Voraussetzungen darstellt, dessen man sich bewusst sein muss, um die eigene wissenschaftliche Praxis kontrollieren zu können. 
Der Kapital-Begriff wird von Bourdieu nicht in einem marxistischen Sinne verwendet, weil er ihn nicht ökonomisch definiert oder andersherum gesagt: das ökonomische Kapital ist nur eine der möglichen Kapitalarten. Schon in der präkapitalistischen Gesellschaft Algeriens hatte er festgestellt, dass das Handeln einer Ökonomie gehorchte, die nicht die einer Geld-Ökonomie war, sondern eine Logik der Ehre. Immer werde das Handeln durch eine Rationalität bestimmt, der die Suche nach Optimierung zugrunde liegt: es gelte hier, die Ehre, das Ansehen zu vermehren. Bourdieu nannte dieses Gut, das die Akteure der präkapitalistischen Gesellschaft anvisierten symbolisches Kapital. Wenn er den Begriff Kapital übernimmt, dann vor allem wegen dessen formalen (und nicht inhaltlichen) Kriterien: immer geht es um Akkumulationsstrategien, um die Transmission eines Erbes, um Gewinnschöpfung.

Der Kapitalbegriff ist für Bourdieu ähnlich wie der Habitus-Begriff mit Geschichtlichkeit verbunden. Die gesellschaftliche Welt stellt nicht bloß einen statischen Gleichgewichtszustand dar, sondern akkumulierte Geschichte. Der Begriff der Kapitalakkumulation führt so die geschichtliche Tiefe in die Analyse ein. Die Kapitalaneignung ist gleichzeitig eine Aneignung von sozialer Energie in Form verdinglichter oder lebendiger Arbeit. Im Kapital ist aber auch eine gewisse Gesetzmäßigkeit angelegt; darum verläuft das gesellschaftliche Leben insbesondere das Wirtschaftsleben, so Bourdieu, nicht wie ein Glücksspiel, d.h. völlig zufällig. Beim Glücksspiel gibt es keine Kontinuität, keine sukzessive Akkumulation oder Vererbung von erworbenen Besitztümern oder Eigenschaften. „Das Kapital ist eine der Objektivität der Dinge innewohnende Kraft, die dafür sorgt, dass nicht alles gleich möglich oder gleich unmöglich ist. Die zu einem bestimmten Zeitpunkt gegebene Verteilungsstruktur verschiedener Arten und Unterarten von Kapital entspricht der immanenten Struktur der gesellschaftlichen Welt, d.h. der Gesamtheit der ihr innewohnenden Zwänge, durch die das dauerhafte Funktionieren der gesellschaftlichen Wirklichkeit bestimmt und über der die Erfolgschancen der Praxis entschieden wird" (Bourdieu 1992a: 50).

Man muss aber den Begriff des Kapitals in allen seinen Erscheinungsformen betrachten. Die Wirtschaftstheorie habe sich ihren (engen) Kapitalbegriff von einer ökonomischen Praxis aufzwingen lassen, die eine historische Erfindung des Kapitalismus sei. Dieser Kapitalbegriff reduziere die gesellschaftlichen Austauschverhältnisse auf den bloßen Warenaustausch, der vom ökonomischen Eigennutz geleitet ist. Alle anderen Formen des sozialen Austauschs erscheinen dann als uneigennützige Beziehungen, die keiner Ökonomie gehorchen. Indem die Wirtschaftswissenschaft zu einer bloßen Wissenschaft der Marktbeziehungen geworden ist, habe sie zugleich die Entstehung einer allgemeinen Wissenschaft der 
Ökonomie der Praxis verhindert. Bourdieu geht es nun gerade darum, eine Ökonomie des Handelns zu entwerfen, was aber nie im engen wirtschaftlichen Sinn gemeint ist. Für ihn gibt es auch im Bereich der sozialen oder kirchlichen Arbeit keine selbstlosen (interessefreien) Akte; ein bestimmter Habitus entfaltet sich hier in einem Feld, in dem Selbstlosigkeit geachtet und somit auch belohnt wird (und sei es bloß durch eine symbolische Belohnung oder durch Gottes Lohn). Durch die religiöse Sprache wird die Ökonomie, die dieser Praxis zugrunde liegt, euphemisiert. Eine wirklich allgemeine Wissenschaft der Praxis muss in der Lage sein, alle die Praxisformen mit ein zu beziehen, die einer Ökonomie gehorchen, aber als solche im gesellschaftlichen Leben nicht erkannt werden oder das Ökonomische im engeren Sinne gerade verneinen. Entgegen den naiv idyllischen Vorstellungen über die vorkapitalistischen Gesellschaften (oder die kulturellen Sphären der kapitalistischen Gesellschaften) ,richten sich die praktischen Handlungen auch dann noch am ökonomischen Kalkül aus, wenn sie, die sie sich der Logik des Interessenkalküls (im eigentlichen Sinne) entziehen und sich an nichtmateriellen und zu quantifizierenden Einsätzen orientieren, den Anschein von Interesselosigkeit vermitteln“ (Bourdieu 1976: 345). Die Theorie der ökonomischen Handlungen im engeren Sinn stellt für Bourdieu nur einen Spezialfall innerhalb einer allgemeinen Theorie der ökonomischen Handlungen dar.

Es ist darum völlig falsch, Bourdieu Ökonomismus zu unterstellen, wie das besonders bei deutschen Kommentatoren der Fall ist, etwa bei Axel Honneth, der Bourdieus Ansatz von reinen Utilitarismen bestimmt sieht (Honneth 1984: 145150); es geht Bourdieu gerade darum, die Vielzahl der Handlungen nicht auf eine wirtschaftliche Logik zu reduzieren, sondern eine Ökonomie im breiten Sinn zu postulieren, die dem Handeln eine Rationalität zuerkennt. Diese Rationalität wird bestimmt durch die formalen Charakteristika, die eine abstrakte Kapitaltheorie herausgearbeitet hat. Diese universelle Dynamik ist in den vier Kapitalarten am Werk, die Bourdieu unterscheidet: a.) das ökonomische Kapital, das unmittelbar in Geld konvertierbar ist (Erbschaft, materielle Güter, Produktionsmittel) und das sich besonders gut für die Institutionalisierung in der Form des Eigentumsrechts eignet; b.) das kulturelle Kapital (die intellektuelle Qualifikation, die man durch das familiäre Milieu mitbekommen hat und das durch schulische Titel institutionalisiert wird); c.) das soziale Kapital (das Kapital an sozialen Verpflichtungen und Beziehungen); d.) das symbolische Kapital (das Ansehen, das mit dem Besitz dieser oder jener Kapitalsorte einhergeht, und das bisweilen in Form von Adelstiteln institutionalisiert wird).

Bourdieu hat sich sehr früh mit der Ökonomie der präkapitalistischen Gesellschaft in Algerien auseinandergesetzt und dabei gezeigt, dass eine Ökono- 
mie immer an historische und soziale Bedingungen gebunden ist. Eine Ökonomie, die auf Treue und Glauben beruht, wie er sie in der Kabylei antraf, legt der ganzen Gruppe Ehrenpflichten auf, die mit dem kalten Gesetz des eigennützigen Kalküls völlig unvereinbar sind. Eine Ökonomie im engeren Sinne, die nur auf geldbasiertem Warenaustausch beruht, ist für ihn historisch gesehen nicht universell. Aber auch in entwickelten Gesellschaften spielt das ökonomische Kapital keine exklusive Rolle.

\section{Das kulturelle Kapital}

In seinen zahlreichen Untersuchungen widmete sich Bourdieu der immensen Bedeutung des kulturellen Kapitals. Er griff bei der Konstruktion des kulturellen Kapitals innerhalb des Bildungssystems auf Max Webers Unterscheidung von „Klassenlage“ (der „Marktlage“ entsprechenden Chancen auf dem Güter- und Arbeitsmarkt) und „Klassenstand“ (der „Stellung“ in der Hierarchie von Ehre und Prestige) zurück. Neben den ökonomischen Unterschieden spielen hier auch symbolische Unterscheidungen eine Rolle, bei denen es nicht mehr bloß um den Besitz von Gütern geht, sondern um die Art, sie zu verwenden und als Mittel der Distinktion einzusetzen. Die Manier, die Form einer Handlung oder der Umgang mit einem Gegenstand, tritt an die Stelle der Funktion: „Daher besitzen von allen Unterscheidungen diejenigen das größte Prestige, die am deutlichsten die Stellung in der Sozialstruktur symbolisieren, wie etwa Kleider, Sprache oder Akzent und vor allem die „Manieren“, Geschmack und Bildung. Denn sie geben sich den Anschein, als handelte es sich um Wesenseigenschaften einer Person, ein aus dem Haben ableitbares Sein, eine Natur, die paradoxerweise zu Bildung, eine Bildung, die zu Natur, zu einer Begnadung und einer Gabe geworden seien" (Bourdieu 1970: 60).

Nicht so sehr oder nicht allein der Besitz ökonomischen Kapitals, sondern der des kulturellen Kapitals macht den entscheidenden Unterschied in der AnsehensHierarchie aus. Bourdieu unterscheidet dabei zwischen drei Formen des kulturellen Kapitals; es kann existieren: 1.) im verinnerlichten, inkorporierten Zustand, in Form von dauerhaften Dispositionen, 2.) in objektiviertem Zustand, in Form von kulturellen Gütern wie Bildern, Büchern, Nachschlagewerken, in denen bestimmte Theorien und Gegentheorien Spuren hinterlassen haben, und schließlich 3.) in institutionalisiertem Zustand in der Form von Stellen und Titeln, die einen besonderen Besitz von kulturellen Kapital offiziell bestätigen. 


\subsection{Inkorporiertes kulturelles Kapital}

Auch hier trennt Bourdieu nicht zwischen Bewusstsein und Körperlichkeit. In seinen Augen ist das kulturelle Kapital auch körpergebunden (,,inkorporiert"); es ist das Produkt einer Verinnerlichung. Der Erwerb der Bildung ist ein Prozess, der Zeit kostet. Diese Zeit muss der einzelne persönlich investieren und kann die Aufgabe nicht delegieren. Der Faktor der Zeit spielt hier eine nicht unwichtige Rolle: die schon in der Primärerziehung erworbene Bildung stellt gewonnene Zeit dar. Die in diesem Kontext nicht erworbene Bildung ist in doppeltem Sinne verloren, weil das Verlorene überdies später nachgeholt werden muss. An der Gesamtdauer des Bildungserwerbs lässt sich so das Volumen des Bildungskapitals in etwa messen. Die in der familiären Primärerziehung und der anschließenden schulischen Sekundärerziehung erworbene Bildung wird zu einem Bestandteil der Person („,den man ihr nicht wegnehmen kann“); dieser verinnerlichte Besitz kann darum im Unterschied zum Geld oder zu Adelstiteln nicht durch Geschenk, Vererbung oder Tausch unmittelbar weitergegeben werden. Indirekt wirkt sich der Besitz von kulturellem Kapital innerhalb einer Familie schon aus; Bourdieu spricht hier von einer hochgradig verschleierten, ja unsichtbaren ,sozialen Vererbung" (Bourdieu 2001: 114). Entscheidend ist auch der Seltenheitswert des jeweiligen kulturellen Kapitals (Hochschulabschluss in einem Land mit niedriger Abiturientenquote, Lesekompetenz in einer Welt von Analphabeten), aus denen sich weitere Extraprofite ziehen lassen. Dieser Seltenheitswert wird erhalten, weil nicht alle Familien über die Mittel verfügen, um ihre Kinder über die obligatorische Schulpflicht hinaus ausbilden zu lassen. So wird auch durch diese Kapitalform Ungleichheit geschaffen, bzw. verstärkt. Die Übertragung von Kulturkapital ist nach Bourdieu die am besten verschleierte Übertragung von Kapital. Wenn die sichtbaren Formen der Übertragung des (ökonomischen) Kapitals sozial missbilligt werden, gewinnen große Investitionen in die individuelle Bildung und Ausbildung an Gewicht, um die bestehenden Verhältnisse aufrecht zu erhalten. Die Verbindung zwischen den ökonomischen und dem kulturellen Kapital stellt die Zeit dar (,Zeit ist Geld“). Man muss über ökonomisches Kapital verfügen, um den Bildungsprozess möglichst früh einsetzen zu lassen und bis zu einem optimalen Abschluss ausdehnen zu können.

\subsection{Objektiviertes kulturelles Kapital}

Objektiviertes kulturelles Kapital - Bücher, Bilder - ist materiell übertragbar wie ökonomisches Kapital. Man kann das materielle Substrat verkaufen; es handelt sich aber um symbolische Güter, deren Wert sich nicht auf den materiellen Wert 
des Substrats reduzieren lässt; diese Güter sind gleichzeitig Bedeutung. Um diese Bedeutung erfassen oder entziffern zu können, muss man über Bildungskapital, d.h. inkorporiertes kulturelles Kapital verfügen. Der Eigentümer muss selbst das für die Aneignung und Nutzung der symbolischen Güter erforderliche inkorporierte Kulturkapital erwerben oder sich auf die Dienste von Inhabern eines solchen Kapitals stützen. Diese Aufgabe kommt oft Intellektuellen zu, die nicht über den ökonomischen Besitz, wohl aber über eine Deutungskompetenz verfügen. Diese sind so gleichzeitig Beherrschte und Herrschende. Nach Bourdieu scheint alles darauf hinzudeuten, dass ,die kollektive Macht der Inhaber von Kulturkapital - und damit auch die für seine Beherrschung erforderliche Qualifikationszeit - zunimmt. Dem steht allerdings entgegen, dass die Inhaber von ökonomischem Kapital (als der dominierenden Kapitalform) die Inhaber von kulturellem Kapital in eine Konkurrenzsituation bringen können" (Bourdieu 2001: 117-118).

Nach Bourdieu darf man aber nicht vergessen, dass das objektivierte Kulturkapital nur dann symbolisch aktiv ist, wenn es von Handelnden angeeignet und genutzt, d.h. interpretiert oder instrumentalisiert wird. Die Partitur wird erst dann zu einem Wert, wenn sie vom Orchester interpretiert, die Skulptur dann, wenn sie ausgestellt oder gedeutet, das Buch dann, wenn es gelesen wird. Die Gewinne richten sich dabei nach der Beherrschung dieses objektivierten Kapitals, folglich nach dem Grad des inkorporierten Kapitals. Weil die Aneignung der Kulturgüter Anlagen und Kompetenzen voraussetzt, die ungleich verteilt sind (obwohl scheinbar angeboren), bilden diese Werke nach Bourdieu ,den Gegenstand einer exklusiven (materiellen oder symbolischen) Aneignung, und weil ihnen die Funktion von (objektiviertem oder inkorporiertem) kulturellen Kapital zukommt, sichern sie einen Gewinn an Distinktion - im Verhältnis zum Seltenheitsgrad der zu ihrer Aneignung notwendigen Instrumente - und einen Gewinn an Legitimität, den Gewinn überhaupt, der darin besteht, sich so, wie man ist, im Recht, im Rahmen der Norm zu fühlen" (Bourdieu 1982: 359). Die anerkannte legitime Kultur wirkt so aufgrund ihrer Distinktionsqualität in Klassengesellschaften als Herrschaftsinstrument, während in klassenindifferenten Gesellschaften Kultur allen zugänglich ist und deshalb nicht diese Funktion ausübt.

\subsection{Institutionalisiertes kulturelles Kapital}

Das institutionalisierte kulturelle Kapital existiert in Form von Titeln und Stellen wie Schul- oder Universitätsabschlüssen. Diese von offiziellen Institutionen verliehenen Ausweise erworbener Bildung verleihen dieser einen juristisch garantierten Wert. Der Titel oder das Examen schafft eine scharfe Grenze zwischen dem, der bestanden hat und dessen kulturelle Kompetenz ein für allemal garan- 
tiert scheint, und dem, der nicht ausgezeichnet (mit andern Worten: stigmatisiert) wurde oder Autodidakt ist und der seine Kompetenz stets unter Beweis stellen muss. „Im krassen Unterschied zu den Inhabern eines kulturellen Kapitals ohne schulische Beglaubigung, denen man immer abverlangen kann, den Beweis für ihre Fähigkeiten anzutreten, da sie nur sind, was sie tun, schlichte Produkte ihrer Leistung, brauchen die Inhaber von Bildungspatenten - ähnlich Trägern von Adelstiteln [...] nur zu sein, was sie sind [...]" (Bourdieu 1982: 48-49). Der Erwerb von schulischen Titeln setzt die Investition von Zeit voraus und damit auch von ökonomischem Kapital, das in kulturelles verwandelt wird, in der Hoffnung, dass sich dieser Prozess wieder umkehrt und der schulische Titel auf dem Arbeitsmarkt materielle und symbolische Gewinne abwirft.

Bourdieu hat die Hypothese des kulturellen Kapitals entwickelt, um die Ungleichheit der schulischen Leistungen von Kindern aus unterschiedlichen sozialen Klassen aus einem sozialen Kontext (unterschiedliche Ausstattung mit kulturellem Kapital) und nicht bloß über die individualistische naturalistische These der Begabung zu erklären. Gleichzeitig richtete er sich gegen den Begriff des Humankapitals, den Ökonomen wie Gary Becker als Reaktion auf den Sputnikschock entwickelt hatten. ${ }^{3}$ Humankapital wurde definiert als Bildungsinvestition (Fähigkeiten und Fertigkeiten sowie das Wissen, das in Personen verkörpert ist und durch Erziehung, Ausbildung, Weiterbildung und Erfahrung erworben werden kann), um ökonomisch verwertbare Tätigkeiten auszuüben. ${ }^{4}$ Bildung und Weiterbildung er-

3 Der Sputnikschock wurde ausgelöst durch den Start des ersten künstlichen Erdsatelliten Sputnik 1 durch die Sowjetunion am 4. Oktober 1957, der die technische Überlegenheit der Sowjetunion im Bereich der Raumfahrt belegte. Das löste eine Krise in der Selbstwahrnehmung Amerikas aus, das sich als technologisch fortschrittlichstes Land verstand. Der Schock löste in den USA eine umfassende Reform des Bildungssystems aus. Ein Hauptaugenmerk lag auf der Förderung bislang bildungsferner Schichten.

4 Gary S. Becker, geb. 1930 war einer der ersten Ökonomen, der die Wirtschaftswissenschaft auf Gebiete ausdehnte, die eher der Soziologie zugewiesen wurden. 1992 wurde ihm der Nobelpreis verliehen. In den sechziger und siebziger Jahren hatte er zusammen mit anderen Autoren das Konzept des Humankapitals in die Wissenschaft eingeführt (Becker 1964). Zu seiner These über den langfristigen Zusammenhang zwischen dem Besuch vorschulischer Betreuungseinrichtungen und dem späteren Erwerbsleben gab es kaum empirische Untersuchungen. Die norwegischen Ökonomen Tarjei Havnes und Magne Mogstad gingen vom norwegischen Kindergartengesetz des Jahres 1975 aus, durch das die staatlich subventionierten Kindergartenplätze annähernd verdreifacht wurden. Sie untersuchten vergleichend 2006 Populationen, die von dem Gesetz profitieren oder eben noch nicht profitieren konnten. Bei den Personen, die eine vorschulische Betreuungseinrichtung besucht hatten, ,sank die Wahrscheinlichkeit, später die Schule abzubrechen. Zudem stieg die Wahrscheinlichkeit deutlich an, später zu studieren. Vor allem Kinder aus bildungsfernen Schichten haben davon profitiert. Schliesslich sank die Wahrscheinlichkeit, später auf staatliche Transferzahlungen angewiesen zu sein.“ (Müller 2011). Bourdieus Kritik an einer einseitigen Ausrichtung auf die ökonomische Rendite der Ausbildung trifft auch auf diese Studie zu. 
scheinen dann nicht mehr als Konsumgüter, sondern als Investitionen, als Produktionsfaktoren ähnlich wie das physische Kapital. Das Humankapital der Mitarbeiter wird dann nicht mehr bloß als Produktions- und Kostenfaktor, sondern als ein Potential gesehen, das zum Unternehmenserfolg und zu volkswirtschaftlichem Wohlstand beiträgt. Bourdieu kritisiert die ökonomistische Ausrichtung dieses Begriffs, den im Übrigen die Gesellschaft für deutsche Sprache zum Unwort des Jahres 2004 erklärte, weil die Menschen dadurch nun mehr als „ökonomisch interessante Größen" verstanden würden. Bourdieu stellt seinerseits fest, die Humankapital-Theorie messe die schulischen Investitionen nur an den Profiten, die sich in Geld ausdrücken lassen. Die Humankapital-Theoretiker würden vor allem die am besten verborgene und sozial wirksamste Bildungsinvestition unberücksichtigt lassen: die „Transmission kulturellen Kapitals in der Familie“ (Bourdieu 2001: 113). Die letztlich ökonomistische Ausrichtung der Humankapital-Theorie übersehe, dass „der schulische Ertrag schulischen Handelns vom kulturellen Kapital abhängt, das die Familie zuvor investiert hat, und dass der ökonomische und soziale Ertrag des schulischen Titels von dem ebenfalls ererbten sozialen Kapital abhängt, das zu seiner Unterstützung zum Einsatz gebracht werden kann“" (Bourdieu 2001: 113).

\section{Soziales Kapital}

Mit dem Begriff soziales Kapital bezeichnet Bourdieu „die Gesamtheit der aktuellen und potentiellen Ressourcen, die mit dem Besitz eines dauerhaften Netzes von mehr oder weniger institutionalisierten Beziehungen gegenseitigen Kennens und Anerkennens verbunden sind [...] es handelt sich dabei um Ressourcen, die auf der Zugehörigkeit zu einer Gruppe beruhen" (Bourdieu 1992a: 63). Es geht hier um ein Prinzip von sozialen Wirkungen, die sich auf der Ebene der individuell Handelnden feststellen lassen, die aber mehr sind als die Summe der individuellen Eigenschaften der Handelnden. Diese Wirkungen werden dann sichtbar, wenn Akteure mit gleichwertiger (ökonomischer oder kultureller Kapitalausstattung) unterschiedliche Profite erzielen, weil sie das Kapital einer Gruppe (Familie, Ehemaligen-Vereinigungen, Clubs) mobilisieren können. Diese Sozialkapitalbeziehungen existieren nur auf der Grundlage von Tauschbeziehungen, die sich stets erneuern. Aus der Zugehörigkeit zur Gruppe ergeben sich sowohl materielle wie symbolische Profite; aus dieser Zugehörigkeit können sich dauerhafte Verpflichtungen ergeben, die auf subjektiven Gefühlen oder auf Rechtsansprüchen beruhen. Das soziale Kapital der Gruppe äußert sich nicht nur in der Förderungs- und Solidaritätsverpflichtung, sondern auch im abgestimmten Ausschluss Gruppen- 
fremder. Das soziale Kapital kann so die Wirkung der beiden andern Kapitalarten bestärken. Bourdieu geht es so immer darum zu ermitteln, über welche (oft verborgenen) Mechanismen die bestehenden Ungleichheiten erhalten oder verstärkt werden. Exemplarisch zeigte er das in seinen Untersuchungen der Eliten in Frankreich auf, die wie kaum anderswo über fest etablierte Assoziationen verfügen, über einen starken Korpsgeist, der über die stark selegierenden Ausbildungswege der Eliteschulen und eine ähnliche soziale Herkunft geschaffen werden. Auch die Manieren (Benehmen, Sprechweise) lassen sich nach Bourdieu in einem gewissen Sinn auch dem Sozialkapital zurechnen, da sie auf unbestimmte Weise auch die Zugehörigkeit zu einer mehr oder weniger angesehenen Gruppe anzeigen.

\section{Die Bedeutung des Sozial- und Kulturkapitals für den Bildungserfolg}

Die entscheidende Bedeutung des (familiären) Kultur- und Sozialkapitals hat Bourdieu vor allem in seinen Untersuchungen zum Bildungswesen oder besser gesagt, des ungleichen Zugangs zur Bildung untersucht. Alle Statistiken belegten einen Zusammenhang zwischen schulischem Erfolg und sozialem Ursprung. Wenn im Jahre 1966 96,6 Prozent der Arbeiterkinder kein Hochschulstudium ergriffen, indes 41,3 Prozent der Kinder aus dem Milieu der Freien Berufe ein Hochschulstudium absolvierten, dann ist der Beruf des Vaters vermutlich nicht der Grund dafür, wohl aber ein Indikator der sozialen Bedingtheit des Zugangs zum (höheren) Bildungswesen. ${ }^{5}$

Mit seinem bildungssoziologischen Analysen, die er zusammen mit JeanClaude Passeron veröffentlichte (Les Héritiers [1964] und La Reproduction [1971] zerstörte Bourdieu den jakobinischen Mythos der per se emanzipatorischen Funktion des Bildungswesens. Frankreich hatte sich mit dem anonymen Aufnahmeverfahren (concours) und der Einführung der allgemeinen Schulpflicht schon relativ früh für ein meritokratisches Prinzip der sozialen Reproduktion entschieden, während in anderen Ländern viel länger eine aristokratische Form der sozialen Reproduktion erhalten blieb ${ }^{6}$. Das französische Bildungssystem führte aber de

$5 \quad$ Diese Situation hat sich auch heute nicht wesentlich verändert. So wird in einer neueren Studie auf der Basis der PISA-Studie nachgewiesen, dass die Schule in Frankreich die soziale Ungleichheit nicht reduziert, sondern noch verstärkt (Siehe Cahuc et al. 2011).

6 So stammten Hochschullehrer in Deutschland am Ende des 19. Jahrhunderts aus einer homogenen sozialen Gruppe. Nach einer vergleichenden Untersuchung von Charle (1990) kamen in Deutschland um die Jahrhundertwende 47,4 Prozent der Professoren aus dem Bereich intellektueller Berufe (Ärzte, Pastoren, Schriftsteller); in Frankreich jedoch bloss 27,9 Prozent. Auch die Zahl der Professoren aus Beamtenfamilien war in Deutschland höher. In Deutschland herrschte wie in den meisten europäischen Ländern die aus der aristokratischen Gesellschaft stammende familieninterne soziale Reproduktion vor (Siehe dazu Charle 1990). 
facto nicht zu einer größeren sozialen Gleichheit, denn das schulische Selektionssystem funktioniert entlang der bestehenden sozialen Hierarchien. Die Aufnahmeprüfungen stehen wohl allen offen, sind aber so aufgebaut, dass die Jugendlichen aus einem kulturell und ökonomisch begünstigten Milieu leichter Erfolg haben.

In seinen Studien ermittelt Bourdieu die entscheidende Bedeutung von familiärer Sozialisation und sozialem Hintergrund. Das scheinbar gerechte Aufnahmeverfahren ignoriert die kulturellen Ungleichheiten, die Kinder unterschiedlicher Klassen voneinander trennen. Die schulische Demokratie setzt eine ökonomische und soziale Demokratie voraus, die so nicht existiert. „Das Schulsystem in seiner gegenwärtigen Form gewährt den Kindern aus den privilegiertesten Milieus ein zusätzliches Privileg, weil die impliziten Werte, die es voraussetzt und vermittelt, die Traditionen, an denen es festhält, ja selbst der Inhalt und die Form der vermittelten und geforderten Bildung, mit den kulturellen Werten und Traditionen der privilegierten Klassen eng verwandt sind" (Bourdieu: 2003: 62).

Die Analysen Bourdieus lieferten den Beweis für die Abhängigkeit des Schulerfolgs vom Bildungsniveau der Eltern ${ }^{7}$. Das kulturelle Erbe werde innerhalb der gebildeten Schichten quasi osmotisch übertragen, so dass die Angehörigen dieser Schichten die Kenntnisse, Fähigkeiten und Einstellungen nicht als das Resultat von Lernprozessen erschienen, sondern als eine Sache der Begabung.

Wie wenig neutral die Bildungsinstitution ist, konnte Bourdieu aus den Ausleseschemata der französischen Universitäten ermitteln. Dort gehe es nicht bloß um die Beherrschung von Inhalten, sondern auch um einen Stil, der letztlich auf das Ideal des Honnête Homme aus dem absolutistischen Frankreich zurückgeht. Heute wie damals sind Umgangsformen wie Ungezwungenheit, Leichtigkeit, Eleganz gefragt, über die nur Jugendliche aus gebildeten Klassen verfügen im Unterschied zum angestrengten und naiven Ernst derjenigen, die aus bildungsferneren Milieus stammen. In Deutschland wurden Kapitel aus den beiden bildungssoziologischen Untersuchungen unter dem Titel Die Illusion der Chancengleichheit (1971) veröffentlicht, was irreführend war, weil Bourdieu nicht die Idee der Chancengleichheit per se als Illusion betrachtete. Er schloss bloß aus den empirischen Untersuchungen, dass Schule und Hochschule in ihrer bestehenden Form den selbstgesetzten Anspruch auf Chancengleichheit nicht einlösten. Der Vorwurf

$7 \quad$ Sacchi et al. (2011) postulierten in einer aktuellen Untersuchung in Anlehnung an Bourdieu aufgrund der grossen Bedeutung der Lehrlingsselektion an der ersten Schwelle auch für die Schweiz starke direkte, an der Schule vorbei wirkende Herkunftseinflüsse. Diese Hypothese wurde voll bestätigt: „Da nachweislich nicht nur die Leistungsbewertung, sondern auch die bewerteten Leistungen von der sozialen Herkunft geprägt sind, sprechen die Befunde auch für starke Einflüsse der sozialen Herkunft auf die Übertrittschancen an der ersten Schwelle. Ganz im Sinne Bourdieus trägt die Schule so dazu bei, vorschulische Herkunftsunterschiede zu perpetuieren" (Sacchi et al. 2011). 
der Resignation und des Fatalismus, den er sich damit einhandelte, war fehl am Platze. Und das umso mehr, da Bourdieu und seine Equipe konkrete Vorschläge entwickelten, um Kindern schon in der Vorschule die kulturellen Erfahrungen zu vermitteln, die Kindern aus begünstigten Klassen von zu Hause mitbringen.

\section{Ein neuer Staatsadel}

Bourdieu hat sich auch später noch bildungssoziologischen Themen gewidmet, so der Struktur des französischen universitären Feldes, dessen spezifische Morphologie gerade in der Zeit der Krise von 1968 sichtbar wird. In Homo Academicus (1984) und in der sehr umfangreichen Untersuchung La Noblesse d'Etat (1989) analysiert er die Elitehochschulen (Grandes Ecoles). Die Führungselite, die aus diesen Schulen hervorgeht, stellt eine Art Oligarchie à la française dar, die Abschlussexamina eine offizielle Legitimierung des Führungsanspruchs des Pariser Großbürgertums. Pierre Bourdieu spricht in diesem Zusammenhang vom neuen Staatsadel. Er ist nicht überzeugt, dass die höhere Anzahl von Universitätsabsolventen zu einer wirklichen Demokratisierung der Eliten führt. Das Bildungssystem trennt mithilfe einer ganzen Reihe von Auslesevorgängen die Besitzer von ererbtem kulturellem Kapital von den Nichtbesitzern. Indem ein Bildungssystem wie in Frankreich eine scharfe Trennung zwischen den Schülern der Grandes Ecoles und den Universitätsstudenten vornimmt, errichtet es soziale Grenzen, die sich nicht sehr unterscheiden von den Grenzen, die einst den Hochadel vom niederen Adel und diesen von den einfachen Nicht-Adligen trennten.

\section{Die sozialen Bedingungen der kulturellen Praktiken}

In der Sammlung von Zeugnissen von Ausgegrenzten, die Bourdieu 1992 unter dem Titel La misère du monde (deutsche Übersetzung: Das Elend der Welt [1997]) veröffentlichte, führt er aus, dass die Vervielfachung der Abiturientenzahlen zu keiner eigentlichen Demokratisierung führe. Die aus den kulturell am stärksten benachteiligten Familien stammenden Schüler oder Studenten erlangten am Ende einer häufig mit schweren Opfern bezahlten Schulzeit oft nur einen entwerteten Titel und wenn sie scheiterten, seien sie zu noch stigmatisierender Ausgrenzung verurteilt. Während die aus gutem Hause stammenden Schüler innerhalb eines sich immer mehr differenzierenden Schulsystems von ihren Familien einen ausgeprägten Platzierungssinn mitbekommen haben und so in der Lage sind, ihre Investitionen in den guten Bildungsgängen und den guten Einrichtun- 
gen zu tätigen, sind „die aus den benachteiligten Familien stammenden Schüler, und ganz besonders die sich meistens ganz und gar selbst überlassenen Einwandererkinder, häufig ab dem Ende der Primarschulzeit dazu gezwungen, sich den Imperativen der Institution Schule oder dem Zufall zu überlassen, um sich ihren Weg durch ein immer komplexeres Universum zu schlagen, und sind aus diesem Grund dazu verurteilt, ein ansonsten äußerst reduziertes kulturelles Kapital falsch und zur Unzeit anzulegen" (Bourdieu 1997: 531).

Die in der Schule vermittelte Kultur ist nicht etwas Neutrales. Sie steht der Kultur der Herrschenden nahe und erhält durch die schulische Vermittlung Legitimität. Kultur ist nicht bloß sozial bestimmt beim Zugang zum Bildungssystem, sondern für die kulturellen Praktiken generell. Hier greift Bourdieu auf den Begriff der Legitimität von Max Weber zurück. Legitimität meint nicht ein objektives Werturteil, sondern den mehr oder weniger hohen Status, den man einer kulturellen Praxis innerhalb einer sozial hierarchisierten Gesellschaft zuschreibt. Das zeigte Bourdieu mit seinen Mitarbeitern in seiner frühen Arbeit über die Fotografie auf, die unter dem bezeichnenden Titel Eine illegitime Kunst erschien. Hier wurde aufgezeigt, dass Fotografie nicht eine neutrale kulturelle Praxis ist, sondern eine sozial bedingte. Für die Angehörigen der Unterschicht gehorcht sie nicht einer Kant'schen Ästhetik des ,interesselosen Wohlgefallens“, sondern folgt einem Interesse; sie soll eine Funktion erfüllen, sie soll gefallen und die Kohärenz der eigenen Gruppe belegen (darum ihre Funktion als Familienfotografie bei Festen oder in den Ferien). Die Vertreter der Mittelschicht bedienten sich auch dieser kulturellen Praktik, wollten sich aber über formale Kriterien vom rein inhaltlichen Fotoverständnis der Leute aus dem einfachen Volk abheben, während Angehörige der Oberschicht sich eher durch den Verzicht auf eine kulturelle Praxis ,auszeichneten“, die allen offen stehe und vor allem inhaltlich bestimmt sei.

Gerade umgekehrt verhält es sich beim Museumsbesuch, dem Bourdieu mit seinem Team 1966 ebenfalls eine Untersuchung widmete (L'amour de l'art). Hier geht es um den ungleichen Zugang zur legitimen Kultur, die wiederum auf eine ungleiche Verteilung des kulturellen Kapitals zurückzuführen ist. Bourdieu wendet sich auch hier wieder gegen die Ideologie eines angeborenen Kunstsinns. Die Werke der Kunst müssen gedeutet und entziffert werden. Die Schlüssel zur Deutung müssen vermittelt und erlernt werden; dies geschieht bei denjenigen, die mit großem (objektiviertem) kulturellen Kapital ausgestattet sind, schon in der Primärsozialisation, was ihnen einen zumindest zeitlichen Vorsprung verschafft.

Die Ideologie der angeborenen intellektuellen Begabung oder des angeborenen Kunstsinns stützt die bestehende soziale Ordnung, d.h. ihre Ungleichheit, die dann als etwas Natürliches erscheint. Das kulturelle Kapital ist aber immer 
ein relationeller Begriff; die einzelnen kulturellen Praktiken oder Objekte haben nicht objektive Bedeutungen, sondern richtet sich nach Maßgabe ihrer sozialen Gebrauchsweise. Jazz kann so durchaus als Distinktionsinstrument und klassische Musik wie die Vier Jahreszeiten von Vivaldi als Hintergrund-Sound im Warenhaus verwendet werden.

Auf der Basis seiner relationistischen Vorstellung der unterschiedlichen Kapitalarten vermochte dann Bourdieu 1979 eine umfassende Sozialstrukturanalyse der französischen Gegenwartsgesellschaft vorzulegen, die auf Deutsch unter dem Titel Die feinen Unterschiede erschien. Das bedeutete einen Bruch mit der marxistischen Sichtweise, die die Gesamtgesellschaft über die letztinstanzliche Determination durch das Ökonomische und die Klassenzugehörigkeit ausschließlich durch den Platz der Akteure im Produktionsprozess bestimmte.

Die soziale Welt wird von Bourdieu als multidimensionaler Raum verstanden, innerhalb dessen Positionen durch Nähe und Entfernungen bestimmt werden. Die Akteure verteilen sich auf der ersten Raumdimension je nach Umfang ihres Kapitals, auf der zweiten je nach der Struktur des Kapitals und auf der dritten Ebene je nach dem Alter des Kapitals (altreich, neureich). Auf einer vertikalen Koordinate lässt sich so das (große oder geringe) Volumen des Gesamtkapitals anzeigen. Auf einer horizontalen Koordinate kann man die Struktur des Kapitals anzeigen (vorwiegend kulturelles oder ökonomisches Kapital). Eine dritte, räumliche Dimension müsste sowohl für die Struktur wie für den Umfang des Kapitals anzeigen, ob es sich um ein Erbe handelt, das weit zurückreicht oder ob es in jüngster Zeit erworben wurde. Gemäß der ersten Dimension stehen die Besitzer eines umfangreichen Gesamtkapitals - etwa Unternehmer, Angehöriger freier Berufe, Hochschullehrer - im Gegensatz zu den mit geringem ökonomischem und kulturellem Kapital ausgestatteten Gruppen. Unter dem Gesichtspunkt der Struktur des Kapitals stehen beispielsweise Hochschullehrer (die reicher an kulturellem als an ökonomischem Kapital sind) im Gegensatz zu den Unternehmern. Die Intellektuellen gehören nach Bourdieu zum Feld der Macht wegen ihres umfangreichen kulturellen Kapitals, aber nicht zu den Herrschenden, weil sie nicht über das entscheidende ökonomische Kapital verfügen; sie sind die „Beherrschten der herrschenden Klasse“, was ihre oft ambivalente Haltung erkläre.

Der Raum der sozialen Positionen, der durch das Volumen, die Struktur und das Alter der verschiedenen Kapitalsorten bestimmt wird, manifestiert sich im Raum der Lebensstile, die sich in den spezifischen kulturellen Praktiken äußern. Denn die Symbolsysteme bringen Herrschaftsbeziehungen zum Ausdruck. Die kulturellen Ausdruckssysteme strukturieren sich in einer Hierarchie, die die kulturelle Legitimität in ihren Abstufungen definiert. Der Sphäre der Legitimität mit 
universalem Anspruch werden klassische Musik, Malerei, Skulptur, Literatur und Theater zugerechnet, die durch Instanzen der Legitimation (wie Universitäten oder Akademien) sanktioniert werden. Am anderen Pol situiert sich die Sphären, die dem individuellen Belieben anheimgestellt sind wie die Inneneinrichtung einer Wohnung, die Kosmetik, die Kleider und die Küche. Hier gibt es bloß illegitime Legitimierungsinstanzen (Werbung, Haute Couture). Zwischen diesen beiden Polen situiert sich die Sphäre der konkurrierenden Legitimationsinstanzen, Praktiken, die weder strengen Regeln noch rein individuellem Geschmack gehorchen: Film, Fotografie, Tanz, Chanson.

Über die Positionen im sozialen Raum (bestimmt über Volumen, Struktur und Alter der Kapitalarten), über die durch die Sozialisation bestimmten Dispositionen (Habitusformen), schließlich über die konkreten Äußerungen durch die unterschiedlichen Lebensstile (bestimmt durch mehr oder weniger legitime Kulturpraktiken) lassen sich die groben Klassenvorstellungen erheblich nuancieren, da in jeder Klassenfraktion ganz unterschiedliche Kapitalkombinationen möglich sind. Über die genannten Unterscheidungskriterien lassen sich bestimmte Klassen konstruieren, die aber für Bourdieu theoretische Klassen bleiben, Produkte eines Klassifizierungsvorganges. Hier liegt eine Bruchstelle zur marxistischen Tradition, die die konstruierte Klasse mit der realen Klasse gleichsetzt oder die, um den Vorwurf von Marx gegenüber Hegel aufzugreifen, die Sache der Logik mit der Logik der Sache identifiziert. Gerade in Gesellschaften, in denen sich 80 Prozent der Mittelklasse zurechnen, wird die Abgrenzung durch unterschiedliche Lebensstile unabdingbar. Es entstehen neue theoretische Klassen, die mit dem alten substantialistischen Klassenschema nichts zu tun haben. Auch in den sowjetischen Ländern, die dem Mythos der klassenlosen Gesellschaft huldigen, bilden sich, wie das Bourdieu noch anlässlich eines Vertrages in Ostberlin im Jahre 1989 ausführte, feine Unterschiede - durch die Partizipation am politischen Kapital, das eine private Nutzung öffentlicher Güter und privilegierten Zugang zur Bildung ermöglicht.

\section{Das symbolische Kapital}

Das symbolische Kapital ist nicht eine weitere Kapitalsorte, sondern sie bezeichnet das Ansehen, das Prestige, die Ehre, die einem der Besitz dieser oder jener Kapitalsorte in einem spezifischen Bereich einbringt; es ist die „wahrgenommene und als legitim anerkannte Form der drei [...] Kapitalien" (Bourdieu 1985: 11). Das symbolische Kapital ist besonders wichtig, weil es die Bedeutung der jeweils anderen Kapitalsorten verstärkt, weil es die Anerkennung durch die Gemein- 
schaft betont. Das institutionalisierte kulturelle Kapital, das durch ein Diplom, einen Studienabschluss belegt wird, kann eine symbolische Macht ausüben, die man nicht allein auf das inkorporierte Kapital des Diplomierten zurückführen kann. Die Hierarchie der Kapitalsorten variiert jedoch von einem Bereich zum anderen; deren Stellenwert ist keineswegs unveränderlich: „Gleich Trümpfen in einem Kartenspiel, determiniert eine bestimmte Kapitalsorte die Profitchancen im entsprechenden Feld (faktisch korrespondiert jedem Feld oder Teilfeld eine Kapitalsorte, die in ihm als Machtmittel und Einsatz im Spiel ist). So bestimmt der Umfang an kulturellem Kapital (Analoges gilt - mutatis mutandis - für ökonomisches Kapital) die Gewinnchancen in den Spielen, in denen kulturelles Kapital wirksam ist, und damit die Stellung innerhalb des sozialen Raums (zumindest insoweit sie vom Erfolg im kulturellen Feld abhängt)“"(Bourdieu 1985: 10). Das symbolische Kapital wahrt seine Wirkkraft nur innerhalb eines spezifischen Feldes und ist darum kaum in ein anderes Feld transferierbar. Das Ansehen etwa, das ein Individuum im politischen Feld genießt, verhilft ihm nicht zu einem analogen Ansehen im literarischen Feld.

Das symbolische Kapital lässt die realen Unterschiede als natürlich, als selbstverständlich erscheinen über einen Prozess der symbolischen Transfiguration. Das Renommee, das Prestige, die Ehre verleihen der Macht eine Art Evidenz. Die Akteure setzen ihr symbolisches Kapital ein, um ihre Sicht der sozialen Welt durchzusetzen. Sie besitzen Macht proportional zum Umfang ihres symbolischen Kapitals, d.h. proportional zum Maß ihrer Anerkennung durch die Gruppe. Das symbolische Kapital verleiht vor allem eine Benennungsmacht, „ein Akt symbolischer Durchsetzung, derweil von einem Mandatsträger des Staates, Inhaber des Monopols über die legitime symbolische Gewalt vollzogen - auf die ganze Stärke des Kollektivs, des Konsens, des common sense bauen kann" (Bourdieu 1985: 23-24). Die Logik der offiziellen Nomination manifestiert sich sehr anschaulich über den Adels-, Bildungs- oder Berufstitel, der als ein institutionalisiertes kulturelles Kapital nicht nur legitim ist, sondern als juristisch abgesicherte Bezeichnung auch legal ist, d.h. ein symbolisches Kapital darstellt. Die Bedeutung dieser Nomination erklärt auch, dass soziale Akteure für eine Position optieren können, die großes Prestige bringt zuungunsten einer Position, die ökonomisch gesprochen rentabler aber weniger prestigereich wäre. „Eine Berufsbezeichnung, ein Titel bildet (wie Lohn oder Gehalt) eine positive oder negative Vergütung, im Sinne einer Unterscheidungsmarke (Emblem oder Stigma), deren Wert sich nach der Stellung innerhalb eines hierarchisch gestaffelten Systems von Titeln richtet und die auf diese Weise zur Festlegung der jeweiligen Positionen von Akteuren und Gruppen beiträgt. Es ist unter derartigen Umständen nur folgerichtig, 
wenn die Akteure auf praktische wie symbolische Strategien zur Maximierung des symbolischen Gewinns von Nominierung und Namensgebung zurückgreifen" (Bourdieu 1985: 25).

\section{Symbolische Gewalt}

Das symbolische Kapital ist dann auch die Basis für das, was Bourdieu symbolische Gewalt nennt. Auf der Basis der Autorität, des Prestiges des Mandatsträgers kann eine Ordnung als legitim, normal, natürlich empfunden werden, wodurch kaschiert wird, dass sie auf Machtverhältnissen beruht. Die symbolische Gewalt ist im Unterschied zur physischen unsichtbar; sie führt dazu, dass sie von den Beherrschten akzeptiert wird, ohne sich dessen bewusst zu sein. Denn im Prozess der Sozialisierung wird diese Ordnung internalisiert. Bourdieu zitiert in diesem Zusammenhang David Hume: „Nichts ist für diejenigen erstaunlicher, die die menschlichen Dinge mit einem philosophischen Auge betrachten, als die Leichtigkeit zu sehen, mit der die Mehrheit (the many) von der Minderheit (the $f e w)$ regiert wird, und die Unterwürfigkeit zu beobachten, mit der die Menschen ihre eigenen Gefühle und Leidenschaften zugunsten ihrer Führer verleugnen“ (Bourdieu 2001a: 228).

Im Unterschied zu Foucault sieht Bourdieu Herrschaft nicht so sehr als Produkt von Disziplinierung oder Dressur, sondern als Folge der symbolischen Gewalt, die darum so wirksam ist, weil sie nicht wahrgenommen wird; es wird nicht wahrgenommen, wie sich die subjektiven Strukturen unbewusst an die objektiven Strukturen anpassen.

Für Bourdieu gibt es zwei Formen der Herrschaft: die eine, die auf nackter Gewalt - der Waffen oder des Geldes - beruht und die symbolische Gewalt, die viel subtiler und weniger sichtbar ist. Beide Formen der Herrschaft schaffen ein Verhältnis der Abhängigkeit, der Unterwerfung, für das es keine Rekursinstanz gibt. Die verkannte symbolische Gewalt äußert sich als Verpflichtung, als Erkenntlichkeit, als Schuldigkeit, der man sich nicht entziehen kann. Nach Bourdieu ist die sanfte Gewalt ein wirkungsvolles Mittel bei der Erziehung der Kinder. Diese werden folgsam wegen der Anerkennung, die man ihnen zollt. Die Suche nach Anerkennung werde so später zu einem sehr starken Antrieb jedes Handelns. Der Soziologe sieht darin die eigentliche Wurzel des menschlichen Drangs nach symbolischem Kapital: nach Ruhm, nach Ehre, nach Ansehen; man suche danach gleichzeitig aus Selbstsucht und wegen der Anerkennung, die man von den anderen erhoffe. Die symbolische Macht setze sich nur darum durch, weil diejenigen, die sie erleiden, ohne sich dessen bewusst zu sein, mitwirken; die Unterordnung 
ist so weder die Folge eines physischen Zwanges noch Ausdruck einer freiwilligen Knechtschaft. Die Machtverhältnisse sind in unserem Körper eingeschrieben und äußern sich in spontanen Gesten, etwa der Ehrerbietung gegenüber demjenigen, den wir als Höherstehenden vermuten oder im Ausdruck der Schüchternheit oder der Angst, im Erröten, in Ungeschicklichkeit - körperliche Reaktionen, die sich aufgrund eines Gefühls der Unterlegenheit auslösen, das wir über unseren Verstand allein nicht meistern können.

Für Bourdieu ist auch die männliche Herrschaft, der er eines seiner letzten Werke widmete, Manifestation einer symbolischen Gewalt. Wenn die meisten französischen (und wohl auch nicht-französischen) Frauen sich einen Lebensgefährten wünschten, der größer und älter sei als sie, dann weil sie den Eindruck vermeiden möchten, sie seien innerhalb des Paares dominant, was sie sozial abwerten würden. Sie würden so unbewusst die äußeren Zeichen einer dominierten Position akzeptieren.

\section{Gibt es eine Aufhebung der Machtverhältnisse?}

Sind alle unsere Beziehungen Machtbeziehungen, sind wir notwendigerweise Herrschende oder Beherrschte? Pierre Bourdieu sieht Möglichkeiten, aus diesem Machtverhältnis auszubrechen, etwa im Bereich der Familie, die als Institution versucht, jedem der Mitglieder auf Dauer Gefühle zu vermitteln, die die Integration aller garantieren. Der Ritus der Übernahme des Familiennamens schaffe ein Zusammengehörigkeitsgefühl und das, was zuerst nur eine Fiktion sei, die auf dem gemeinsamen Familiennamen beruhe, könne zu einer realen Gruppe werden, die durch affektive Bindungen geeint sei.

Ist aber Liebe zwischen den Partnern möglich ohne symbolische Gewalt? Beruht sie nicht stets auf akzeptierter Beherrschung oder Unterwerfung, die verkannt und damit anerkannt wird? Die Mythen der Verkörperung der Frau oder des Mannes zeigten von Eva bis Cleopatra Frauen, die durch ihre Liebe eine geheimnisvolle Macht ausüben oder Männer à la Don Juan, die sich als Jäger oder Eroberer darstellen. Und doch, so betont Pierre Bourdieu zu Recht, bedeutet eine Beziehung der Liebe oder der Freundschaft, dass Macht und Machtverhältnisse aufgebrochen werden, dass die Beherrschung beherrscht, die symbolische Gewalt ausgeschlossen werden können. Dieser Bruch mit der alltäglichen Machtordnung vollzieht sich aber nicht auf einen Schlag. Durch ein stetes Bemühen, ohne jede Berechnung, kann ein Raum der Liebe entstehen, ein Bereich ohne Gewalt, der Beziehungen ermöglicht, die auf reiner Gegenseitigkeit beruhen, die eine Art gegenseitiger Anerkennung ermöglichen, so dass ein jeder sich in seiner Existenz 
gerechtfertigt fühlt, wie Sartre sagte, und zwar in seinen noch so zufälligen eigenen Zügen. Die Zufälligkeit einer zufälligen Begegnung wird so zu etwas Absolutem. Der französische Schriftsteller Montaigne brachte das in einer einmaligen Formel auf den Punkt. „Wenn man mich fragte, warum er mein Freund war”, so bemerkte er in Bezug auf seinen größten Freund La Boétie, „dann würde ich bloß sagen: weil er es war, weil ich es war". Diese Welt der Uneigennützigkeit macht Beziehungen möglich, die nicht instrumentalisiert sind, die auf dem Glück beruhen, Glück zu schenken und im Staunen des anderen unerschöpfliche Gründe zu finden, selber zu staunen. Die Liebe gehorcht so einer Ökonomie des Austausches, deren höchste Form die Hingabe ist, die jeder Form des Marktes widerspricht, auch der des Arbeitsmarktes, dessen Akteure austauschbar und nicht einmalig sind. Die reine Liebe ist so nach Pierre Bourdieu eine historisch noch relativ junge Erscheinung, ähnlich wie die reine Kunst - l'art pour l'art-, die in ihrer Absolutheit durchaus etwas mit der reinen Liebe zu tun hat.

Auf den Schlussseiten der Meditationen findet sich eine intensive und auch sehr persönliche Reflexion über das symbolische Kapital. Bourdieu knüpft auch hier wieder an Blaise Pascal an. Die einzige Gewissheit des Lebens ist nach Pascal die Kontingenz. Die Menschen täten alles, um diese Endlichkeit zu vergessen, stürzten sich ins Vergnügen oder in die Gesellschaft. Jedoch: „Jeder stirbt allein“. Der Mensch erscheint als ein Wesen ohne Seinsgrund, das vom tiefen Bedürfnis nach Rechtfertigung erfüllt ist: „Das Elend des Menschen ohne Gott“. Bourdieu interpretiert dieses Pascal-Wort. Neben Gott erscheine die Gesellschaft als einzige Instanz, die die menschliche Existenz zu rechtfertigen vermöge. Die soziale Welt verfüge über die sozusagen göttliche Macht, den Menschen der Kontingenz und der Unverbindlichkeit zu entreißen. Eine gesellschaftliche Aufgabe zu haben, erwartet, gefragt zu werden, für die anderen zu zählen, stellt eine fortwährende Rechtfertigung der Existenz dar. Der Soziologe belegt dies ex negativo aus der frühen Untersuchung Durkheims über die Motive des Selbstmordes. „Die soziale Welt vergibt das seltenste Gut überhaupt: Anerkennung, das heißt ganz einfach Daseinsberechtigung." (Bourdieu 2001a: 309) Diese Anerkennung ist für Bourdieu das symbolische Kapital, dessen Verteilung durchaus ungleich ist; die Hierarchie der Anerkennung ist keineswegs gleichbedeutend mit derjenigen, die durch Macht und Reichtum konstituiert wird. Jede Kapitalart (ökonomisches, soziales, kulturelles Kapital) kann zum symbolischen Kapital werden oder besser eine symbolische Wirkung erzielen, wenn sie explizite oder implizite Anerkennung verschafft. Indem es Macht-Beziehungen in Sinn-Beziehungen verwandelt, vermag das symbolische Kapitel den einzelnen aus der Bedeutungslosigkeit zu entreißen. 
Bekannt und anerkannt zu sein bedeutet auch, selber anerkennen zu können. Die' gesellschaftlichen Institutionsriten, die über ein Diplom oder einen Ausweis eine Kompetenz, eine öffentliche Rolle oder schlicht die bloße Identität offiziell bestätigen, anerkennen, dass der einzelne das ist, was er zu sein vorgibt. Durch diese Zeichen wird er zum vollgültigen Mitglied der Gruppe, der Gemeinschaft und hat Anteil an der Ewigkeit der Gruppe, welche die Existenz der einzelnen überdauert. „Die Einsetzungsriten geben ein vergröbertes, besonders deutliches Bild vom Wirken der Institution, dieses willkürlichen Wesens, das die Macht hat, von Willkür zu befreien und die Daseinsberechtigung schlechthin zu verleihen, die Bestätigung nämlich, dass ein kontingentes, von Krankheit, Gebrechen und Tod angreifbares Wesen der transzendenten, unsterblichen Würde so würdig ist wie die soziale Ordnung, der es angehört" (Bourdieu 2001a: 315). Nach Bourdieu legitimiert so die Gesellschaft über ihre Instanzen, die offiziell nennen, ernennen und auszeichnen, den einzelnen, und die Soziologie wird dergestalt zu einer Art Theologie der letzten Rechtfertigung.

\section{Literatur}

Becker, Gary. 1964. Human Capital: A Theoretical and Empirical Analysis, with Special Reference to Education. London: The University of Chicago Press.

Bourdieu, Pierre. 1970/1974. Zur Soziologie der symbolischen Formen. Frankfurt a. M.: Suhrkamp. Bourdieu, Pierre. 1976. Entwurf einer Theorie der Praxis auf der ethnologischen Grundlage der kabylischen Gesellschaft. Frankfurt a. M.: Suhrkamp.

Bourdieu, Pierre. 1982. Die feinen Unterschiede. Kritik der gesellschaftlichen Urteilskraft. Frankfurt a. M.: Suhrkamp.

Bourdieu, Pierre. 1985. Sozialer Raum und ,Klassen'. Leçon sur la leçon. Zwei Vorlesungen. Frankfurt a. M.: Suhrkamp.

Bourdieu, Pierre. 1992. Rede und Antwort. Frankfurt a.M.: Suhrkamp.

Bourdieu, Pierre. 1992a. Die verborgenen Mechanismen der Macht, Hrsg. Margareta Steinrücke, Hamburg: VSA.

Bourdieu, Pierre. 1997. Das Elend der Welt. Zeugnisse und Diagnosen alltäglichen Leidens in der Gesellschaft. Konstanz: UVK.

Bourdieu, Pierre. 2001. Wie die Kultur zum Bauern kommt. Über Bildung, Schule und Politik, Hrsg. Margareta Steinrücke. Hamburg: VSA.

Bourdieu, Pierre. 2001a. Meditationen. Zur Kritik der scholastischen Vernunft. Frankfurt a. M.: Suhrkamp. 
Bourdieu, Pietre. 2003. Interventionen 1961-2001. Sozialwissenschaft und politisches Handwerk. Band I. Hamburg: VSA.

Cahuc, Pierre, Stéphane Carcillio, Olivier Galland und André Zylbergerg. 2011. La Machine à trier. Comment la France divise sa jeunesse. Paris: Eyrolles.

Charle, Christophe. 1990. Naissance des , intellectuels' 1880-1900. Paris: Les Editions de Minuit.

Honneth, Axel. 1984. Die zerrissene Welt der symbolischen Formen. Zum kultursoziologischen Werk Pierre Bourdieus. Kölner Zeitschrift für Soziologie und Sozialpsychologie 36: 145-150. Jurt, Joseph. 2008. Bourdieu. Stuttgart: Reclam.

Jurt, Joseph. 2010. Die Habitus-Theorie von Pierre Bourdieu. LiThes. Zeitschrift für Literatur- und Theatersoziologie 3: 5-13. Elektronische Version http://lithes.uni-graz.at/lithes/10_03.html (Stand: 30.11 .2011$)$

Müller, Matthias. 2011. Kinderbetreuung in Norwegen. Der Ausbau der frühkindlichen Bildung wirkt. Neue Zürcher Zeitung, 247, 22. Oktober.

Sacchi, Stefan, Sandra Hupka-Brunner, Barbara E. Stalder und Markus Gangl. 2011. Die Bedeutung von sozialer Herkunft und Migrationshintergrund für den Übertritt in anerkannte nachobligatorische Ausbildungen in der Schweiz. In: Transitionen im Jugendalter: Ergebnisse der Schweizer Längsschnittstudie TREE, Hrsg. Manfred Max Bergman, Sandra Hupka-Brunner, Anita Keller, Thomas Meyer, Barbara S. Stalder, 120-147. Zürich: Seismo.

Wacquant, Loïc.1995. Durkheim et Bourdieu : le socle commun et ses fissures. Critique 51: 646-660. 